\title{
A STRONG NONCOMMUTATIVE ERGODIC THEOREM
}

\author{
BY E. CHRISTOPHER LANCE
}

Communicated by R. G. Bartle, June 5, 1976

Let $\alpha$ be an automorphism of a von Neumann algebra of such that 20 has a faithful $\alpha$-invariant normal state $\rho$. For $A$ in $\mathcal{U}$ and $n=1,2, \ldots$, write

$$
s_{n}(A)=\frac{1}{n}\left[A+\alpha(A)+\alpha^{2}(A)+\cdots+\alpha^{n-1}(A)\right] .
$$

The result referred to in the title is the following.

TheOREM 1. For each $A$ in 20 and $\epsilon>0$ there exist an $\alpha$-invariant element $\hat{A}$ and a projection $E$ in or such that $\rho(E)>1-\epsilon$ and

$$
\left\|\left(s_{n}(A)-\hat{A}\right) E\right\| \rightarrow 0 \text { as } n \rightarrow \infty \text {. }
$$

We describe the convergence by saying that $s_{n}(A) \rightarrow \hat{A}$ "almost uniformly."

Consider the case where $\mathscr{U}$ is commutative. Then there is a probability measure space $(X, S, \mu)$ such that $\mathscr{U}$ is isomorphic to $L^{\infty}(X, \mu)$ in such a way that $\rho$ corresponds to the functional $f \longmapsto \int_{X} f d \mu$. Also, $\alpha$ corresponds to an automorphism of $L^{\infty}(X, \mu)$ which is induced by an invertible measure-preserving transformation of $X$. It is easy to see (using Egorov's theorem) that almost uniform convergence in $\mathcal{X}$ corresponds to almost everywhere convergence on $X$. So in this setting the above theorem reduces to the classical pointwise ergodic theorem of G. D. Birkhoff [1] (for a bounded function $f$ ).

The first ingredient in the proof of Theorem 1 is the theorem of Kovács and Szücs [2], which establishes the existence of the conditional expectation mapping $A \mapsto \hat{A}$, and can be adapted to show that $s_{n}(A) \rightarrow \hat{A}$ strongly. This result can be regarded as a noncommutative version of the mean ergodic theorem. Next, it is necessary to show the existence of a large set of elements $A$ in $\mathscr{O}$ for which we can take the projection $E$ to be the identity.

THEOREM 2. Let $\mathfrak{U}_{u}=\left\{A \in \mathfrak{U}:\left\|_{s_{n}}(A)-\hat{A}\right\| \rightarrow 0\right\}$. For any $A$ in $\mathfrak{U}$ there is a bounded sequence $\left(A_{k}\right)$ in $\mathfrak{U}_{u}$ which converges strongly to $A$.

The proof of Theorem 2 involves some Fourier analysis and the spectral theorem for a unitary operator. If we write $B_{k}=A-A_{k}$ then $B_{k} \rightarrow 0$ strongly and so $s_{n}\left(B_{k}\right) \rightarrow 0$ strongly as $k \rightarrow \infty$, for each $n$. If we knew that this convergence was, in a suitable sense, uniform in $n$, we could complete the proof of Theorem 1 by von Neumann algebra techniques. The desired uniformity is provided by the following theorem.

AMS (MOS) subject classifications (1970). Primary 46L10; Secondary 28A65, 46A05, $47 \mathrm{A35}$. 
THEOREM 3. If $A$ is a positive element in the unit ball of 20 and $\rho(A)=\delta$ then there is a positive element $C$ of 20 with $\|C\| \leqslant 2$ such that $\rho(C) \leqslant 4 \delta^{1 / 2}$ and $s_{n}(A) \leqslant C$ for all $n$.

The intuitive content of Theorem 3 is that if $A$ is positive and its expected value $\rho(A)$ is sufficiently small, then all its averages $s_{n}(A)$ are fairly small. In the commutative case, this is more or less the content of the maximal ergodic theorem, which appears to be an essential ingredient in the proof of the pointwise ergodic theorem. To prove Theorem 3, we reduce it (using the correspondence between the ideal structure of a $C^{*}$-algebra and the facial structure of its state space) to a statement about the state space of a $C^{*}$-algebra which is a special case of the following result about compact convex sets.

THEOREM 4. Let $K$ be a compact convex set in a real locally convex space, $\alpha$ a bicontinuous affine automorphism of $K, f$ a continuous real-valued affine function on $K, z$ a point of $K$ with $\alpha(z)=z$ and $n$ a positive integer. Then either $f(z) \geqslant$ 0 or there exist a face $F$ of $K$ and points $z_{1}$ in $F, z_{2}$ in $K$ such that $z$ is a convex combination $\lambda z_{1}+(1-\lambda) z_{2},(1-\lambda) f\left(z_{2}\right) \geqslant 0$ and $s_{m}(f) \leqslant 0$ on $F$ for $1 \leqslant m$ $\leqslant n$.

In the above, $s_{m}(f)=\left[f+\alpha f+\cdots+\alpha^{m-1} f\right] / m$, where $\alpha f$ is the affine function given by $\alpha f(x)=f(\alpha(x))(x \in X)$. The proof of Theorem 4 involves some Choquet theory and an adaptation of one of the standard proofs of the maximal ergodic theorem.

Detailed proofs of these results will be published elsewhere.

\section{REFERENCES}

1. G. D. Birkhoff, Proof of the ergodic theorem, Proc. Nat. Acad. Sci. U.S.A. 17 (1931), 656-660.

2. I. Kovács and J. Szücs, Ergodic type theorems in von Neumann algebras, Acta Sci. Math. (Szeged) 27 (1966), 233-246. MR 35 \#753. ENGLAND

DEPARTMENT OF MATHEMATICS, MANCHESTER UNIVERSITY, MANCHESTER, 